\title{
Reduction in Hospitalizations for Respiratory Diseases during the First COVID-19 Wave in Greece
}

\author{
Christos Kyriakopoulos $^{\mathrm{a}}$ Athena Gogali ${ }^{\mathrm{a}}$ Konstantinos Exarchos ${ }^{\mathrm{a}}$ Dimitrios Potonos ${ }^{\mathrm{a}}$ \\ Konstantinos Tatsis $^{a}$ Vasiliki Apollonatou ${ }^{b}$ Stelios Loukides ${ }^{b}$ Spyros Papiris ${ }^{b}$ loanna Sigala ${ }^{c}$ \\ Paraskevi Katsaounou $^{c}$ Maximos Aggelidis ${ }^{d}$ Evangelika Fouka ${ }^{d}$ Konstantinos Porpodis ${ }^{d}$ \\ Theodoros Kontakiotis $^{d}$ Fotis Sampsonas ${ }^{\mathrm{e}}$ Theodoros Karampitsakos ${ }^{\mathrm{e}}$ Argyris Tzouvelekis ${ }^{\mathrm{e}}$ \\ Eleni Bibaki ${ }^{f}$ Konstantinos Karagiannis ${ }^{f}$ Katerina Antoniou ${ }^{f}$ Nikolaos Tzanakis $^{f} \quad$ Ilias Dimeas $^{g}$ \\ Zoe Daniilg $^{g}$ Konstantinos Gourgoulianis ${ }^{9}$ Maria Kouratzi $^{\text {h }}$ Paschalis Steiropoulos ${ }^{i}$ \\ Emmanouil Antonakis ${ }^{j} \quad$ Ilias C. Papanikolaou ${ }^{j} \quad$ Georgios Ntritsos ${ }^{k} \quad K^{\prime}$ Konstantinos Kostikas ${ }^{a}$ \\ a Respiratory Medicine Department University of Ioannina Faculty of Medicine, loannina, Greece; ${ }^{\text {b }}$ 2nd Respiratory \\ Medicine Department University of Athens Attikon Hospital, Athens, Greece; ${ }^{c} 1$ st Dept of Critical Care and \\ Pulmonary Medicine National and Kapodistrian University of Athens, Evangelismos General Hospital, Athens, \\ Greece; ${ }^{d}$ Respiratory Medicine Department Aristotle University of Thessaloniki Faculty of Health Sciences, General \\ Hospital of Thessaloniki G Papanikolaou, Thessaloniki, Greece; ${ }^{e}$ Respiratory Medicine Department University of \\ Patra School of Health Sciences, University General Hospital of Patra, Patra, Greece; fDepartment of Respiratory \\ Medicine Faculty of Medicine, University of Crete, Crete, Greece; ${ }^{9}$ Department of Respiratory Medicine University

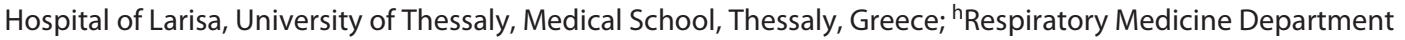 \\ Democritus University of Thrace Department of Medicine, University General Hospital of Alexandroupolis, \\ Alexandroupolis, Greece; 'Respiratory Medicine Medical School of Alexandroupolis, Democritus University \\ of Thrace, Thrace, Greece; 'Corfu General Hospital, Respiratory Medicine Department Corfu General Hospital, \\ Respiratory Medicine Department Corfu, Ionian Islands, Corfu, Greece; ${ }^{k}$ University of loannina, Department of \\ Hygiene and Epidemiology, loannina, Greece
}

\section{Keywords}

Severe acute respiratory syndrome coronavirus-2 . COVID-19 • Hospital admissions · First pandemic wave . Health services

\begin{abstract}
Introduction: During the first COVID-19 wave, a considerable decline in hospital admissions was observed worldwide. Aim: This retrospective cohort study aimed to assess if there were any changes in the number of patients hospitalized for respiratory diseases in Greece during the first CO-
\end{abstract}

VID-19 wave. Methods: In the present study, we evaluated respiratory disease hospitalization rates across 9 tertiary hospitals in Greece during the study period (March-April 2020) and the corresponding period of the 2 previous years (20182019) that served as the control periods. Demographic data and discharge diagnosis were documented for every patient. Results: Of the 1,307 patients who were hospitalized during the study period, 444 (35.5\%) were males with a mean $( \pm S D)$ age of $66.1 \pm 16.6$ years. There was a 47 and $46 \%$ reduction in all-cause respiratory morbidity compared to the corresponding periods of 2018 and 2019, respectively. The mean incidence rate for respiratory diseases during the karger@karger.com

www.karger.com/res
(C) 2021 S. Karger AG, Basel
Correspondence to:

Konstantinos Kostikas, ktkostikas@gmail.com

Karger 
study period was 21.4 admissions per day, and this rate was significantly lower than the rate during the same period in 2018 (40.8 admissions per day; incidence rate ratio [IRR], $0.525 ; 95 \%$ confidence interval $[\mathrm{Cl}], 0.491-0.562 ; p<0.001)$ or the rate during 2019 (39.9 admissions per day; IRR, 0.537; $95 \% \mathrm{Cl}, 0.502-0.574 ; p<0.001)$. The greatest reductions (\%) in the number of daily admissions in 2020 were observed for sleep apnoea ( $87 \%$ vs. 2018 and $84 \%$ vs. 2019) followed by admissions for asthma (76\% vs. 2018 and $79 \%$ vs. 2019) and chronic obstructive pulmonary disease (60\% vs. 2018 and $51 \%$ vs. 2019), while the lowest reductions were detected in hospitalizations for pulmonary embolism (6\% vs. 2018 and $23 \%$ vs. 2019) followed by tuberculosis ( $25 \%$ vs. both 2018 and 2019). Discussion/Conclusion: The significant reduction in respiratory admissions in 2020 raises the reasonable question of whether some patients may have avoided seeking medical attention during the COVID-19 pandemic and suggests an urgent need for transformation of healthcare systems during the pandemic to offer appropriate management of respiratory diseases other than COVID-19.

(c) 2021 S. Karger AG, Basel

\section{Introduction}

The novel severe acute respiratory syndrome coronavirus-2 (SARS-CoV-2), which is the coronavirus disease (COVID-19) causing agent, is a highly infectious viral pathogen that is accountable for the ongoing pandemic [1]. Regardless of COVID-19 incidence among different countries, recommendations on strict social containment/self-confinement led to unprecedented changes in daily life worldwide. At the same time that the global health community was focussing on bringing the spread of COVID-19 under control, with almost all of the countries instantly reorganizing their healthcare systems, concerns were brought up about public health collateral damage. Reports from countries highly impacted by the pandemic, such as Italy [2] and the UK [3], suggested a considerable decline in acute coronary syndrome-related hospitalization rates and primary percutaneous coronary intervention admissions. Meanwhile, epidemiological findings from countries with low COVID-19 incidence during the first wave of the pandemic, including Greece, were comparable [4]. During this same period, in Norway, a $29 \%$ reduction in the weekly number of admissions for stroke and $41 \%$ for transient ischemic attack during the 1 st 7 weeks of the pandemic lockdown were observed [5], while admissions for heart failure in Italy were reduced by $70 \%$ [6]. In the USA alone, there was an overall
$42.8 \%$ reduction in admissions, varying from $23.7 \%$ for pancreatitis to $68.6 \%$ for chronic obstructive pulmonary disease (COPD)/asthma [7]. Possible explanations for such phenomena include the patients' reluctance to seek medical care and more conservative management by healthcare systems. Last spring, we also observed a remarkable reduction in the incidence of acute respiratory diseases $[8,9]$. In the present study, we evaluate the rates of admissions in respiratory medicine departments in Greece during the 1st wave of the COVID-19 pandemic and compare them to the corresponding period of the 2 previous years.

\section{Methods}

We conducted a multicentre retrospective observational cohort study in 9 respiratory medicine departments of tertiary hospitals in Greece, in which we evaluated hospital admissions for the most common causes of respiratory disease, namely, COPD, lung cancer, asthma, pulmonary embolism, sleep apnoea, tuberculosis, interstitial lung disease (ILD), lower respiratory tract infections (other than COVID-19), fibreoptic bronchoscopy/fine needle biopsy (FOB/FNB), or other causes (including but not limited to haemoptysis, pleural effusion, fever, or pulmonary oedema). Data were retrospectively collected for the period of March 1st to April 30th during the COVID-19 outbreak in 2020 and the corresponding 8-week control period in 2018 and 2019. The study was approved by the Ethics Committee of the University Hospital of Ioannina.

The 1st confirmed case of COVID-19 in Greece was identified on February 26th, 2020, and by March 1st, 2020, there were only 7 confirmed cases. During the 9-week period of our study, starting on March 1st, the Greek government authorities gradually enforced various measures: (1) March 10th, 2020: closure of all schools and universities and strict recommendation of self-confinement behaviour; (2) March 16th, 2020: lockdown of $80 \%$ of all business activities; and, finally, (3) March 23rd, 2020: drastic measures confining free mobility which were imposed until May 3rd, 2020. Until April 30th, 2020, there were a total of 2,605 confirmed COVID-19 cases and 140 deaths in Greece [10].

During the study period (March-April 2020), the average daily COVID-19 incidence, throughout Greece, varied from 4 cases per day (March 1st, 2020) to 93 cases per day (April 2nd, 2020), declining to 18 cases per day on April 30th, 2020. From the 2,598 total confirmed cases with SARS-CoV-2, during the corresponding period, 1,885 were admitted, mainly in hospitals that were transformed into COVID-19 hospitals [10]. The hospitals, in which the study has held, received limited COVID-19 admissions and continued their regular operation. Except for polysomnography studies that were constrained in some centres, other elective and semielective procedures (including FOB, CT-guided needle biopsy, or thoracoscopy) were available in these hospitals.

We compared hospitalization rates between the onset of the COVID-19 outbreak period in Greece (March 1st to April 30th, 2020) and the 2 corresponding periods of 2019 and 2018, as well as the mean hospitalization rates. For each admission diagnosis, 
Table 1. Demographics and clinical characteristics of patients in the COVID-19 (2020) and the 2 control periods (2018 and 2019)

\begin{tabular}{lccc}
\hline Characteristic & $2018(N=2,487)$ & $2019(N=2,434)$ & $2020(N=1,307)$ \\
\hline Age, mean (SD), years & $67.74(15.94)$ & $67.62(15.4)$ & $66.07(16.58)$ \\
Female gender, $n(\%)$ & $808(35.5)$ & $835(36.7)$ & $444(35.55)$ \\
COPD, $n(\%)$ & $363(14.2)$ & $302(12.5)$ & $146(11.2)$ \\
Cancer, $n(\%)$ & $202(7.8)$ & $199(8.1)$ & $126(9.7)$ \\
Asthma, $n(\%)$ & $96(4.1)$ & $112(4.7)$ & $23(1.8)$ \\
Pulmonary embolism, $n(\%)$ & $47(1.9)$ & $57(2.3)$ & $44(3.4)$ \\
Sleep apnoea, $n(\%)$ & $448(18)$ & $365(14.9)$ & $56(4.2)$ \\
Tuberculosis, $n(\%)$ & $24(0.9)$ & $24(0.9)$ & $18(1.4)$ \\
ILD, $n(\%)$ & $95(4.1)$ & $119(4.9)$ & $26(2)$ \\
Infection, $n(\%)$ & $566(23.1)$ & $551(22.7)$ & $437(33.4)$ \\
FOB/FNB, $n(\%)$ & $126(4.9)$ & $134(5.6)$ & $72(5.5)$ \\
Other, $n(\%)$ & $520(21)$ & $571(23.4)$ & $359(27.4)$
\end{tabular}

FOB/FNB, fibreoptic bronchoscopy/fine needle biopsy; COPD, chronic obstructive pulmonary disease; ILD, interstitial lung disease.

we calculated incidence rates (IRs) by dividing the number of cumulative admissions by the number of days for each time period, and, in addition, using Poisson regression models, we calculated the incidence rate ratio (IRR) for the COVID-19 period against the control periods. The alpha level of 0.05 was used to determine statistical significance. All statistical tests were 2-tailed. All analyses were done with Stata 14.0 (StataCorp LP, College Station, TX).

\section{Results}

Of the 1,307 patients who were hospitalized during the study period, $444(35.5 \%)$ were males with a mean $( \pm \mathrm{SD})$ age of $66.1 \pm 16.6$ years. Of these patients, $146(11.2 \%)$ presented with COPD, 126 (9.7\%) with cancer, $23(1.8 \%)$ with asthma, $44(3.4 \%)$ with pulmonary embolism, 56 (4.2\%) with sleep apnoea, 18 (1.4\%) with tuberculosis, 26 (2\%) with ILD, 437 (33.4\%) with infection, 72 (5.5\%) to undergo FOB/FNB, and 359 (27.4\%) with various other diagnoses. The number of admissions, total and for each respiratory reason in 2018-2020, is presented in Table 1 and Figure 1.

There was a 47 and $46 \%$ reduction in all-cause respiratory morbidity compared to the corresponding periods of 2018 and 2019, respectively. The mean IR for respiratory diseases during the study period was 21.4 admissions per day. This rate was significantly lower than either the rate during the same period in 2018 (total number of admissions, 2,487; 40.8 admissions per day; IRR, 0.525; $95 \%$ confidence interval $[\mathrm{CI}], 0.491-0.562 ; p<0.001)$ or the rate during 2019 (total number of admissions, 2,434; 39.9 admissions per day; IRR, 0.537 ; 95\% CI, 0.502-0.574; $p<$ 0.001 ), as well as to the mean hospitalization rate of 2018 and 2019 (40.3 admissions per day; IRR, 0.531; 95\% CI, $0.497-0.568 ; p<0.001)$.

The greatest reductions (\%) in the number of daily admissions in 2020 were observed for sleep apnoea ( $87 \%$ vs. 2018 and $84 \%$ vs. 2019) followed by admissions for asthma (76\% vs. 2018 and 79\% vs. 2019$)$ and COPD (60\% vs. 2018 and 51\%; vs. 2019), while the lowest reductions were detected in hospitalizations for pulmonary embolism $(6 \%$ vs. 2018 and $23 \%$ vs. 2019$)$ followed by tuberculosis ( $25 \%$ vs. both 2018 and 2019). Admissions for other respiratory infections were also remarkably reduced (23\% vs. 2018 and $20 \%$ vs. 2019). The number of admissions per day and the IRR were lower in 2020 than in either 2018 or 2019 for each of the aforementioned diseases $(p<0.001)$, with the exceptions of pulmonary embolism (IRR, 0.936; $95 \%$ CI, $0.621-1.412 ; p=0.753$ vs. 2018 and IRR, 0.771; $95 \%$ CI, $0.521-1.144 ; p=0.197$ vs. 2019$)$ and tuberculosis (IRR, 0.75; 95\% CI, $0.407-1.382 ; p=0.356$ vs. both 2018 and 2019) (Table 2).

\section{Discussion/Conclusion}

In this analysis from tertiary hospitals in Greece, we observed a significant decrease in respiratory disease hospitalization rates during the 1st wave of the COVID-19 pandemic. The documented reduction differed among diagnoses, the greatest being detected in admissions for 
Table 2. Number of admissions for various respiratory diseases in 2018, 2019, and 2020

\begin{tabular}{llll}
\hline Diagnosis & Study period (IR) & Control periods (IR, IRR [95\% CI]; $p$ value) \\
\cline { 2 - 4 } & $2020(N=1,307)$ & $2018(N=2,487)$ & $2019(N=2,434)$ \\
\hline Total admissions & 21.4 & $40.8,0.525(0.491-0.562) ;<0.0001$ & $39.9,0.537(0.502-0.574) ;<0.0001$ \\
COPD & 2.4 & $5.9,0.402(0.332-0.487) ;<0.0001$ & $4.9,0.483(0.397-0.589) ;<0.0001$ \\
Cancer & 2.1 & $3.3,0.624(0.499-0.779) ;<0.001)$ & $3.3,0.633(0.506-0.791) ;<0.001$ \\
Asthma & 0.4 & $1.6,0.239(0.152-0.378) ;<0.0001$ & $1.8,0.205(0.131-0.321) ;<0.0001)$ \\
Pulmonary embolism & 0.7 & $0.8,0.936(0.621-1.412) ; 0.753$ & $0.9,0.771(0.521-1.144) ; 0.197$ \\
Sleep apnoea & 0.9 & $7.3,0.125(0.095-0.165) ;<0.0001$ & $5.9,0.153(0.116-0.203) ;<0.0001)$ \\
Tuberculosis & 0.3 & $0.4,0.750(0.407-1.382) ; 0.356$ & $0.4,0.750(0.407-1.382) ; 0.356$ \\
ILD & 0.4 & $1.5,0.274(0.177-0.422) ;<0.0001$ & $1.9,0.218(0.143-0.334) ;<0.0001$ \\
Infection & 7.2 & $9.3,0.772(0.681-0.875) ; 0.0003$ & $9.0,0.793(0.404-0.715) ; 0.0001$ \\
FOB/FNB & 1.2 & $2.1,0.571(0.428-0.763) ; 0.0001$ & $2.2,0.537(0.551-0.717) ;<0.0001$ \\
Other & 5.9 & $8.5,0.690(0.604-0.790) ;<0.0001$ & $9.4,0.629(0.502-0.574) ;<0.0001$ \\
\hline
\end{tabular}

The study period was defined as the time between March 1 and April 30, 2020, and the 2 control periods were the corresponding times in 2018 and 2019. CI, confidence interval; IR, incidence rate (number of daily admissions); IRR, incidence rate ratio; COPD, chronic obstructive pulmonary disease; ILD, interstitial lung disease; FOB/FNB, fibreoptic bronchoscopy/fine needle biopsy.

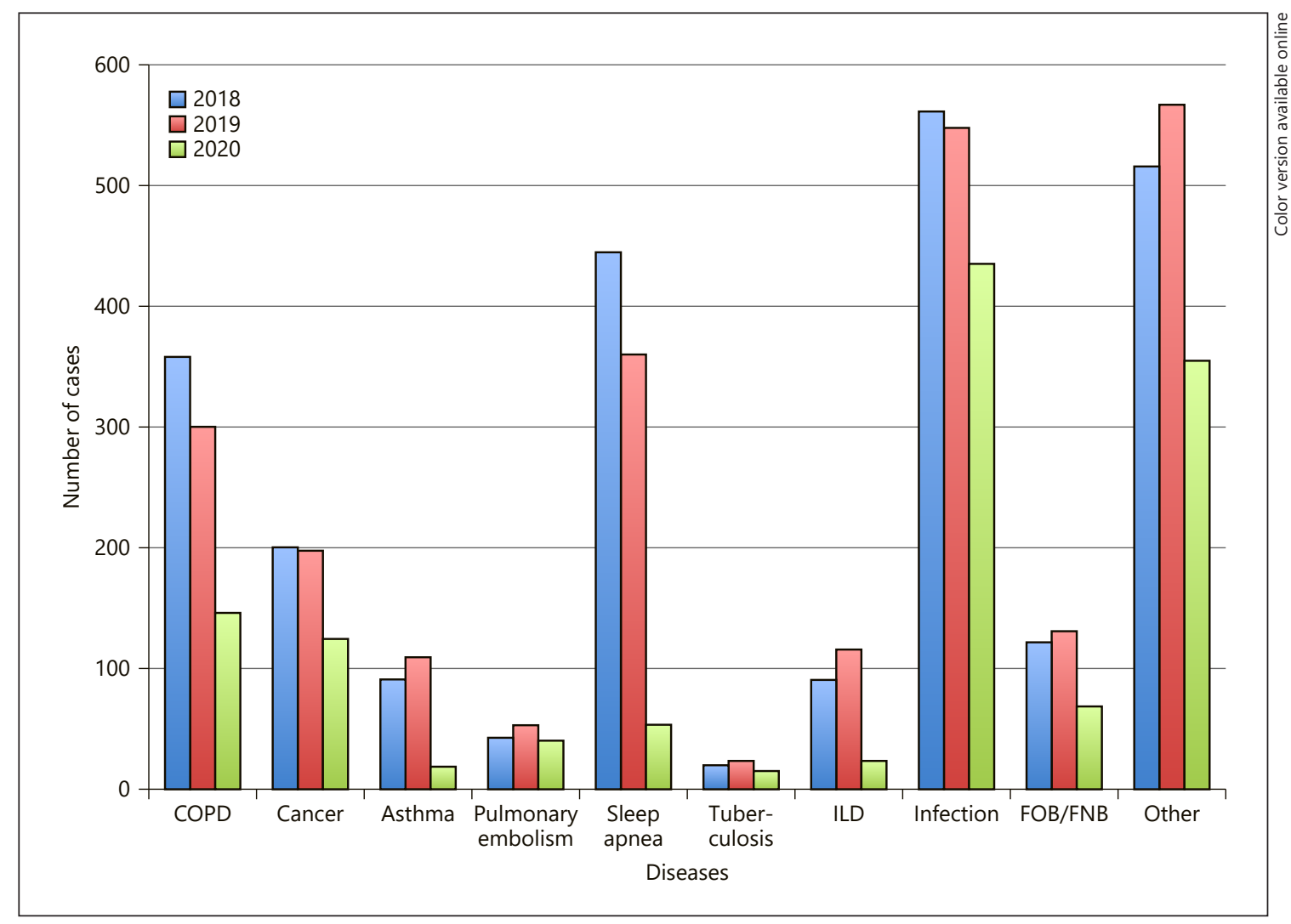

Fig. 1. Number of admissions for various respiratory diseases in 2018, 2019, and 2020. COPD, chronic obstructive pulmonary disease; ILD, interstitial lung disease; FOB/FNB, fibreoptic bronchoscopy/fine needle biopsy. 
sleep apnoea and the lowest for pulmonary embolism, suggesting variable patient and physician behaviours which depend on the nature and the emergency of the disease (postponement of scheduled sleep studies vs. sustained emergency visits due to life-threatening symptoms).

Interestingly, hospitalizations for airway diseases (asthma and COPD) were significantly reduced, a finding that may reflect reduced exposure to aggravating factors due to self-containment, effective management in primary care, or even avoidance to visit hospitals due to the fear of COVID-19 infection. Additionally, the lockdown of all business activities and drastic measures confining free mobility which were imposed led to a concomitant reduction in air pollution and improvement of air quality index $[11,12]$, and this may have had an impact on the reduction in exacerbations of chronic airway diseases. Hospitalizations due to infectious diseases were noticeably reduced, indicating the decreased spread of other viruses such as influenza or RSV or even bacterial pathogens, possibly due to self-confinement, social distancing, and extensive use of facial mask and other protective measures. Hospitalizations due to subacute respiratory symptoms requiring investigation (lung cancer and ILD) were markedly reduced, plausibly due to the patients' hesitance to request medical assistance. The fact that we did not observe a statistically significant reduction on admissions for tuberculosis (18 for 2020 vs. 24 for both 2018 and 2019) may be partially attributed to the small incidence of the disease and also to the fact that the majority of the cases were likely attributed to secondary tuberculosis that would not be affected considerably by social distancing.

Recent data suggest a marked increase in mortality during the 1st wave of the pandemic that was not fully explained by COVID-19 cases alone in Europe [13] and worldwide [14]. The total mortality for the corresponding 9 -week period that we evaluated in the present analysis in Greece was 21,815 deaths, marginally higher than that in
2018 (773 deaths; 3.54\%) and 2019 (395 deaths; 1.81\%) [15]. We cannot draw firm conclusions regarding respiratory mortality as we do not have access to robust data on the causes of death in the areas that we have evaluated in the analysis.

Overall, the observed reduction in respiratory admissions during the 1st COVID-19 wave in Greece raises the reasonable question of whether some patients avoided hospitalization or may have even died from various causes without seeking medical attention during the COVID-19 pandemic. Our data suggest that there is an urgent need for the transformation of healthcare systems during the pandemic to offer appropriate management of respiratory disorders other than COVID-19.

\section{Statement of Ethics}

This article does not contain any studies with human participants or animals performed by any of the authors. It is a multicentre retrospective observational cohort study using epidemiological data. The study was approved by the Institutional Board of the University Hospital of Ioannina (Nr 02325/12-06-2020).

\section{Conflict of Interest Statement}

The authors declare that they have no conflict of interest.

\section{Funding Sources}

The authors received no funding for this study.

\section{Author Contributions}

All authors contributed equally to this study. All authors edited the manuscript and approved the final version.

\section{References}

1 Zhou P, Yang XL, Wang XG, Hu B, Zhang L, Zhang W, et al. A pneumonia outbreak associated with a new coronavirus of probable bat origin. Nature. 2020;579(7798):270-3.

2 De Filippo O, D’Ascenzo F, Angelini F, Bocchino PP, Conrotto F, Saglietto A, et al. reduced rate of hospital admissions for acs during covid-19 outbreak in northern italy. $\mathrm{N}$ Engl J Med. 2020 Jul;383(1):88-9.
3 Mafham MM, Spata E, Goldacre R, Gair D, Curnow $\mathrm{P}$, Bray $\mathrm{M}$, et al. COVID-19 pandemic and admission rates for and management of acute coronary syndromes in England. Lancet. 2020 Aug;396(10248):381-9.

4 Papafaklis MI, Katsouras CS, Tsigkas G, Toutouzas K, Davlouros P, Hahalis GN, et al. "Missing" acute coronary syndrome hospitalizations during the COVID 19 era in Greece: Medical care avoidance combined with a true reduction in incidence?. Clinical Cardiology. 2020;43(10):1142-9.
5 Kristoffersen ES, Jahr SH, Thommessen B, Rønning OM. Effect of COVID-19 pandemic on stroke admission rates in a Norwegian population. Acta Neurol Scand. 2020 Dec; 142(6):632-6. Epub 2020 Jul 14. PMID: 32620027; PMCID: PMC7361547.

6 Severino P, D'Amato A, Saglietto A, D'Ascenzo F, Marini C, Schiavone M, et al. Reduction in heart failure hospitalization rate during coronavirus disease 19 pandemic outbreak. ESC Heart Fail. 2020 Oct. 
7 Birkmeyer JD, Barnato A, Birkmeyer N, Bessler R, Skinner J. The Impact of the COVID-19 Pandemic on Hospital Admissions in the United States. Health Aff. 2020 Sep. 101377hlthaff202000980.

8 Tan JY, Conceicao EP, Sim XYJ, Wee LEI, Aung MK, Venkatachalam I. Public health measures during COVID-19 pandemic reduced hospital admissions for community respiratory viral infections. J Hosp Infect. 2020 Oct;106(2):387-9.

9 Nourazari S, Davis SR, Granovsky R, Austin R, Straff DJ, Joseph JW, et al. Decreased hospital admissions through the emergency de- partment during the COVID-19 pandemic. The American Journal of Emergency Medicine. 2020.

10 National Public Health Organization . Daily report on epidemiological surveillance for new coronavirus disease (COVID-19).

11 Greece . Air pollution country fact sheet. 2020. Sep [cited 2021 Jan 9]. Available from. https://www.eea.europa.eu/themes/air/country-fact-sheets/2020-country-fact-sheets/ greece

12 Greece Air Quality Index (AQI) and Air Pollution information. [cited 2021 Jan 9]. Available from. https://www.iqair.com/greece
13 Vestergaard LS, Nielsen J, Richter L, Schmid D, Bustos N, Braeye T, et al. Excess all-cause mortality during the COVID-19 pandemic in Europe - preliminary pooled estimates from the EuroMOMO network, March to April 2020. Euro Surveill. 2020 Jul;25(26)

14 Bilinski A, Emanuel EJ. COVID-19 and Excess All-Cause Mortality in the US and 18 Comparison Countries. JAMA. 2020.

15 ELSTAT. http//www.statistics.gr, Statistics > Population and Social Conditions $>$ Demography. [cited 2021 Jan 9]. Available from. https:// www.statistics.gr/documents/20181/69e36dbc1604-a320-3cb2-5bb198b1f4cf 